\title{
https://doi.org/10.1007/s10357-020-3716-z
}

\section{Das Klimaschutzgesetz des Bundes - neue Ansätze für den Naturschutz?}

\author{
Juliane Albrecht
}

(c) Der/die Autor(en) 2020

Im vorliegenden Beitrag ${ }^{1}$ sollen speziell die Bezüge und ggf. neue Ansätze des Klimaschutzgesetzes für den Naturschutz herausgearbeitet und bewertet werden. Anknüpfungspunkte für den Naturschutz bieten vor allem die Sektoren Landwirtschaft und Landnutzung. Schwerpunkte bilden dabei die Senkung des Stickstoffeintrages, der Ausbau des Ökolandbaus, Humuserhalt und Humusaufbau im Ackerland, der Schutz von Mooren, der Erhalt von Dauergrünland sowie die klimaangepasste Bewirtschaftung der Wälder. Die hierzu im Klimaschutzprogramm 2030 der Bundesregierung genannten Maßnahmen werden nachfolgend erläutert sowie im Hinblick auf ihre (rechts-) instrumentelle Umsetzung aus Naturschutzsicht bewertet.

\section{Bezüge zwischen Klimaschutz und Naturschutz}

Am 18. Dezember 2019 ist das Bundes-Klimaschutzgesetz (KSG) in Kraft getreten. ${ }^{2}$ Damit wurde in Deutschland auf Bundesebene erstmals ein Klimaschutzgesetz mit verbindlichen Klimaschutzzielen eingeführt. Zur Erreichung der Klimaschutzziele sieht das Gesetz die Aufstellung eines Klimaschutzprogramms und einen Nachsteuerungsmechanismus bei Zielverfehlung vor, enthält Monitoring-Pflichten, normiert die Schaffung eines unabhängigen Sachverständigenrats und schreibt die Vorbildwirkung der Bundesverwaltung fest. Die Umsetzung der im Klimaschutzprogramm enthaltenen Maßnahmen erfordert die Änderung verschiedener Fachgesetze sowie weiterer Maßnahmen (z. B. finanzielle Förderung, Öffentlichkeitsarbeit, Beratung etc.). Aus Naturschutzsicht stellt sich angesichts der anhaltenden Biodiversitätsverluste die Frage, ob sich aus den gesetzlich geregelten Klimazielen und den Instrumenten zu ihrer Erreichung Synergieeffekte ergeben, die zugleich den Anliegen des Naturschutzes verstärkte politische Schubkraft geben können. Grundsätzliche Bezüge zwischen Klimaschutz und Naturschutz bestehen insbesondere im Hinblick auf Klimaschutz-Maßnahmen in den Sektoren Landwirtschaft und Landnutzung (LULUCF). Beiden wirken sich ,,direkt und deutlich auf die Artenvielfalt und die Ökosystemleistungen aus" (12. Erwägungsgrund LULUCF-Verordnung). Ziel des vorliegenden Beitrages ist es, die Bezüge des KSG zum Naturschutz herzustellen und mögliche Ansätze für den Naturschutz herauszuarbeiten und zu bewerten. Letztere ergeben sich dabei nur mittelbar aus dem KSG selbst, sondern aus den im Klimaschutzprogramms 2030 festgelegten Maßnahmen, die wiederum auf den im KSG festgelegten Zielen und den Vorgaben des Klimaschutzplans 2050 beruhen. Demgemäß werden die relevanten Maßnahmen aus den Bereichen Landwirtschaft und Landnutzung aus $\mathrm{Na}$ turschutzsicht identifiziert und behandelt (s. Kap. 2 und 3). Am Ende des Beitrags erfolgt eine zusammenfassende Bewertung aus der Perspektive des Naturschutzes (Kapitel 4).

\section{Naturschutzrelevante Maßnahmen des Sektors Landwirtschaft}

Der Landwirtschaftssektor trägt einen Anteil von 7,4\% an den gesamten THG-Emissionen in Deutschland. Im Jahr

Dr. Juliane Albrecht,

Leibniz-Institut für ökologische Raumentwicklung,

Dresden, Deutschland
2014 betrugen diese 72 Mio. t CO,-̈̈quivalent. Bis 2030 müssen die THG-Emissionen auf 58 Mio. $\mathrm{CO}_{2}$-Äq. gemindert werden (vgl. Anlage 2 KSG). Die größten Emissionsquellen sind die Lachgasemissionen ${ }^{3}$ als Folge des Stickstoffeinsatzes bei der Düngung (25 Mio. t $\mathrm{CO}_{2}$-Äq.), verdauungsbedingte Methan-Emissionen aus der Tierhaltung (25 Mio. t $\mathrm{CO}_{2}$-Äq.), Methan- und Lachgas-Emissionen aus der Wirtschaftsdüngerlagerung ${ }^{4}\left(10 \mathrm{Mio}\right.$. $\mathrm{CO}_{2}$ Äq.) sowie $\mathrm{CO}_{2}$-Emissionen aus dem Kraftstoffverbrauch landwirtschaftlicher Fahrzeuge (6 Mio. t $\left.\mathrm{CO}_{2}-\ddot{A} q.\right){ }^{5} \mathrm{Als}$ Maßnahmen, die einen näheren Bezug zum Naturschutz aufweisen, werden im Klimaschutzprogramm 2030 v.a. die Senkung der Stickstoffüberschüsse und der Ausbau des Ökolandbaus genannt.

\subsection{Senkung der Stickstoffüberschüsse}

Der Einsatz von Stickstoffdüngern zählt zu den wichtigsten Ursachen der THG-Emissionen aus der Landwirtschaft. Ihre Anwendung ist verbunden mit direkten $\mathrm{N}_{2} \mathrm{O}$-Emissionen (Lachgas) aus den gedüngten Böden, indirekten $\mathrm{N}_{2} \mathrm{O}$-Emissionen, die eine Folge des Austrags reaktiver Stickstoffverbindungen wie Nitrat und Ammoniak sind, sowie Emissionen, die im Zuge der Herstellung von Industrie-Dünger (sog. Stickstoff-Mineraldünger) und des Transports der Düngemittel auftreten." 6 Ein wichtiger Pfad zum Erreichen des Klimaschutzziels ist es daher, Stickstoffüberschüsse abzubauen und dauerhaft zu reduzieren. Dies wirkt sich nicht nur günstig auf den Klimaschutz, sondern auch auf den Naturschutz bzw. die Biodiversität aus. Denn Stickstoff ist zwar ein essenzieller Nährstoff für alle Lebewesen. Im Übermaß führt er aber zu Belastungen von Land- und Wasser-Ökosystemen und gefährdet die Artenvielfalt. ${ }^{7}$

Die im Klimaschutzprogramm 2030 aufgeführten Maßnahmen zur Reduzierung des Stickstoffeintrags sind vielfältig. Insoweit wird insbesondere die konsequente Umsetzung der Düngeverordnung (DüngeV) durch die Länder und die Evaluierung der Stoffstrombilanz bis Ende 2021 und deren Weiterentwicklung mit verpflichtender Einführung auf weiteren Betrieben ab dem Jahr 2021 genannt. $^{8}$ Hierzu soll das Düngegesetz (DüngeG) geändert werden. Zudem ist die schrittweise Ausrichtung

1) Vgl. auch den Beitrag: „Albrecht, Das Klimaschutzgesetz des Bundes - Hintergrund, Regelungsstruktur und wesentliche Inhalte“.

2) Bundes-Klimaschutzgesetz vom 12. Dezember 2019 (BGB1. I S. 2513).

3) Lachgas entsteht aus dem Abbau stickstoffhaltiger Verbindungen; LfU, Lachgas, https://www.lfu.bayern.de/klima/klimaschutz/ treibhausgase/lachgas/index.htm (Zugriff: 16.2.2020).

4) Hierbei handelt es sich um organischen Dünger, d.h. Gülle und Mist.

5) Klimaschutzprogramm 2030 der Bundesregierung zur Umsetzung des Klimaschutzplans 2050, Stand: 8.10.2019, S. 104

6) Weingarten et al., Klimaschutz in der Land- und Forstwirtschaft sowie den nachgelagerten Bereichen Ernährung und Holzverwendung. Gutachten, November 2016, S. 161 und 326.

7) Stein-Bachinger/Haub/Gottwald, Biodiversität im Meer und an Land, https://doi.org/10.2312/eskp.2020.1.4.7, S. $108 \mathrm{ff}$.

8) Klimaschutzprogramm 2030 (Fn. 5), S. 108. 
der Gesamtbilanz des Stickstoffüberschusses ${ }^{9}$ am Ziel der Nachhaltigkeitsstrategie von 70 Kilogramm Stickstoff pro Hektar im Jahr $2030^{10}$ als Maßnahme aufgeführt. ${ }^{11}$ Bei Überschreitung der zulässigen Bilanzwerte soll ein Beratungs- und Sanktionierungsmechanismus in Gang gesetzt werden. Zu den weiteren Maßnahmen zählt die Auflegung eines Bundesprogramms Nährstoffmanagement, durch welche die Belastungen der Landwirte im Zusammenhang mit der Änderung des Düngerechts abgemildert werden sollen. ${ }^{12}$

Hintergrund dieser Vorgaben ist das EuGH-Urteil vom 21. Juni $2018^{13}$, in dem ein Verstoß Deutschlands gegen die Nitratrichtlinie $(91 / 676 / \mathrm{EWG})^{14}$ festgestellt wurde. Die Richtlinie verpflichtet die Mitgliedstaaten dazu, die Verunreinigung der Gewässer durch Nitrat aus landwirtschaftlichen Quellen, insbesondere durch Düngung, zu bekämpfen. ${ }^{15}$ Nicht zuletzt auf Grund des eingeleiteten Vertragsverletzungsverfahrens hatte Deutschland im Jahr 2017 die Düngegesetzgebung durch das sog. Düngepaket novelliert. Dieses umfasst Änderungen des DüngeG ${ }^{16}$ und der DüngeV ${ }^{17}$ sowie die Einführung einer sog. Stoffstrombilanzverordnung (StoffBilV) ${ }^{18}$ auf der Grundlage von $\$ 11 \mathrm{a}$ Abs. 2 DüngeG. Letztere enthält nähere Vorschriften über die nach $\$ 11$ a Abs. 2 DüngeG zu erstellende betriebliche Stoffstrombilanz im Hinblick auf die gute fachliche Praxis beim Umgang mit Nährstoffen (Saldo aus Nährstoffzufuhr und -abfuhr). Hierdurch sollen Nährstoffverluste in die Umwelt so weit wie möglich vermieden werden. Im Ergebnis des Urteils befand die EU-Kommission allerdings das Düngepaket 2017 als unzureichend. ${ }^{19}$ Die DüngeV wird daher derzeit erneut überarbeitet, um den Anforderungen der EU-Kommission nachzukommen. ${ }^{20}$

Ein weiterer Maßnahmenbereich betrifft die Senkung der Ammoniak-Emissionen. Ammoniak ist ein indirekt klimarelevantes Gas, da es nach Emission in die Umwelt vielfältigen Umwandlungsprozessen, u. a. zu Lachgas, unterliegt. ${ }^{21}$ Atmosphärische Stickstoff-Depositionen tragen wiederum zur Versauerung und Eutrophierung von Böden und Ökosystemen bei, was langfristig zu einem Nährstoffungleichgewicht, schlechterem Pflanzenwachstum und Verlust an biologischer Vielfalt führen kann. ${ }^{22} \mathrm{Als}$ Maßnahme zur Senkung der Ammoniak-Emissionen wird im Klimaschutzprogramm eine zügige und umfassende Umsetzung aller Minderungsoptionen der Maßnahmen des nationalen Luftreinhalteprogramms zur Erfüllung der National Emission Ceilings Directive (NEC-Richtlinie, 2016/2284/EU) ${ }^{23}$ genannt. ${ }^{24}$ Die Richtlinie schreibt Emissionsreduktionsverpflichtungen für bestimmte Luftschadstoffe, u.a. Ammoniak, vor. Zudem soll ein Investitions- und Förderprogramm zur Unterstützung der notwendigen betrieblichen Anpassungen aufgelegt und fortgeschrieben werden..$^{25}$

Als weiterer Maßnahmenbereich zur Senkung der Stickstoffüberschüsse wird eine gezielte Verminderung von Stickstoffemissionen aus landwirtschaftlichen Böden genannt (z.B. durch Optimierung der zeitlichen Verteilung der Düngung und emissionsarme Düngetechnologien). Schließlich soll der Anteil gasdicht gelagerter Gülle aus der Rinder- und Schweinehaltung auf 70 Prozent erhöht werden. ${ }^{26}$

\subsection{Ausbau des Ökolandbaus}

Die Ausweitung der ökologischen bewirtschafteten Landwirtschaftsflächen, insbesondere des Ökolandbaus gemäß der EU-Ökoverordnung ${ }^{27}$, kann ebenfalls klimaschützend wirken. Denn der Ökolandbau orientiert sich mit seiner Bewirtschaftungsweise an der Nachhaltigkeit (Wirtschaften mit der Natur). Durch eine Umstellung auf Ökolandbau können sich die Lebensgemeinschaften auf den Wirtschaftsflächen erholen, so dass der Ökolandbau auch aus Naturschutz- bzw. Biodiversitätssicht grundsätzlich för- derlich ist. ${ }^{28}$ Der Verzicht auf den Einsatz von Mineraldünger im Gegensatz zur konventionellen Landwirtschaft führt dauerhaft zu THG-Einsparungen. Die Förderung der Kohlenstoffbindung im Boden, ein erhöhter Anteil an Leguminosen $^{29}$ in der Fruchtfolge, die vergleichsweise niedrige Viehbesatzdichte und Grünlanderhalt durch Weidezwang sind weitere Gründe dafür, dass die flächenbezogenen THG-Emissionen im ökologischen Landbau in der Regel niedriger ausfallen als im konventionellen Landbau. ${ }^{30}$ Auf-

9) Der Stickstoffüberschuss beschreibt die Differenz zwischen landwirtschaftlicher Stickstoffzufuhr (z. B. durch Düngemittel, Futtermittel, Saat- und Pflanzgut, Einträge aus der Atmosphäre) und -abfuhr (durch tierische und pflanzliche Produkte). 2015 lag der Wert bei $94 \mathrm{~kg} /$ ha pro Jahr; UBA, Nährstoffeinträge aus der Landwirtschaft und Stickstoffüberschuss; https://www.umweltbundesamt.de/daten/land-forstwirtschaft/naehrstoffeintraegeaus-der-landwirtschaft\#stickstoffuberschuss-der-landwirtschaft (Zugriff: 16.2.2020).

10) Bundesregierung, Deutsche Nachhaltigkeitsstrategie, Neuauflage 2016, S. 65.

11) Klimaschutzprogramm 2030 (Fn. 5), S. 108.

12) Im November 2019 beschloss der Haushaltsausschuss des Bundestages hierfür den finanziellen Rahmen. Bis 2022 sollen finanzielle Mittel in Höhe von 70 Mio Euro zur Förderung für Gülleaufbereitung, Transport und Lagerung an die Landwirte fließen.

13) EuGH, Urt. v. 21.6.2018 - C-543/16, ECLI:EU:C:2018:481.

14) ABl. EG, L 375, 31.12.1991, S. 1.

15) Für Grundwasser gilt ein Nitratgrenzwert i.H.v. $50 \mathrm{mg} / 1$; vgl. Anhang I A Nr. 2.

16) Düngegesetz vom 9.1.2009 (BGB1. I S. 54, 136), zuletzt geändert durch Art. 1 des Gesetzes vom 5.5.2017 (BGBl. I S. 1068).

17) Düngeverordnung vom 26.5.2017 (BGBl. I S. 1305).

18) Verordnung über den Umgang mit Nährstoffen im Betrieb und betriebliche Stoffstrombilanzen vom 14.12.2017 (BGBl. I S. 3942; 2018 I S. 360).

19) Nach Auffassung der EU-Kommission haben die gesetzlichen Maßnahmen von 2017 die vom EuGH festgestellten Mängel (unzureichende Vorschriften zur Begrenzung der Ausbringung von Düngemitteln, zusätzliche Maßnahmen für verseuchte Gebiete, Sperrzeiten und Düngung auf stark geneigten landwirtschaftlichen Flächen) nicht vollständig behoben, https://ec.europa. eu/commission/presscorner/detail/de/inf_19_4251 (Zugriff: 16.2.2020).

20) Zum weiteren Fortgang des Verfahrens s. BMEL, Vertragsverletzungsverfahren, https://www.bmel.de/DE/Landwirtschaft/Pflanzenbau/Ackerbau/_Texte/Duengung.html (Zugriff: 16.2.2020).

21) Weingarten et al. (Fn. 6), S. 61.

22) So gehören z. B. mehr als $70 \%$ der Pflanzenarten, die in Deutschland in der Roten Liste aufgeführt sind, zu den Arten nährstoffarmer Standorte. Im Fall eines Überangebotes an Stickstoff sind diese wegen Verdrängung durch Stickstoff liebende Arten gefährdet. LfU, Eutrophierung und Versauerung, https://www.lfu. bayern.de/luft/schadstoffe_luft/eutrophierung_versauerung/index.htm (Zugriff: 16.2.2020).

23) Richtlinie (EU) 2016/2284 vom 14.12.2016 über die Reduktion der nationalen Emissionen bestimmter Luftschadstoffe, zur Änderung der Richtlinie 2003/35/EG und zur Aufhebung der Richtlinie 2001/81/EG, AB1. EU, L 344, 17.12.2016, S. 1.

24) Klimaschutzprogramm 2030 (Fn. 5), S. 108.

25) Klimaschutzprogramm 2030 (Fn. 5), S. 109.

26) Klimaschutzprogramm 2030 (Fn. 5), S. 109.

27) Verordnung (EG) Nr. 834/2007 des Rates vom 28. Juni 2007 über die ökologische/biologische Produktion und die Kennzeichnung von ökologischen/biologischen Erzeugnissen, ABl. EG, L 189, 20.7.2007, S. 1. Diese wird mit Wirkung zum 1. Januar 2021 durch die Verordnung (EU) Nr. 2018/848, ABl. EU, L 150, 14.6.2018, S. 1 ersetzt.

28) Klimaschutzprogramm 2030 (Fn. 5), S. 114; Stein-Bachinger/ Haub/Gottwald (Fn. 7), 109.

29) Leguminosen (auch bezeichnet als Hülsenfrüchtler) besitzen auf Grund spezieller Bakterien in den Wurzelknöllchen die Fähigkeit zur Bindung von Luftstickstoff im Boden; https://www. spektrum.de/lexikon/geowissenschaften/leguminosen/9452 (Zugriff: 16.2.2020).

30) Weingarten et al. (Fn. 6), S. 192. 
grund der teilweise deutlich niedrigeren Erträge und Leistungen des Ökolandbaus gilt dies allerdings nicht für die produktbezogenen THG-Emissionen, die je nach Standort, Betriebsausrichtung und Management sehr unterschiedlich sind. ${ }^{31}$ Daher wird eine Ausdehnung des Ökolandbaus aus Klimaschutzsicht v.a. dann befürwortet, wenn zusätzliche Voraussetzungen wie z.B. leichte Böden oder ein hoher Grünlandanteil erfüllt und Synergieeffekte mit weiteren Umweltleistungen wie dem Natur- oder Gewässerschutz zu erwarten sind. ${ }^{32} \mathrm{Um}$ den Anteil ökologisch bewirtschafteter Landwirtschaftsflächen zu steigern will die Bundesregierung ,die Rechtsvorschriften zugunsten besonders umweltfreundlicher Verfahren wie dem ökologischen Landbau oder anderer besonders nachhaltiger Verfahren der Landbewirtschaftung weiterentwickeln und die rechtliche und finanzielle Förderung optimieren“". ${ }^{33}$ Dementsprechend werden der Ausbau der Förderung des Ökolandbaus und die Sicherstellung der notwendigen Fördergelder für die Umstellung in den Länderhaushalten und auf Bundesebene als Maßnahmen genannt. ${ }^{34}$ Auch will die Bundesregierung die Zukunftsstrategie Ökologischer Landbau ${ }^{35}$ in der Praxis verankern, um zusätzliche Wachstumsimpulse entlang der gesamten Wertschöpfungsketten zu setzen. Hierdurch werde der ökologische Landbau indirekt gefördert. ${ }^{36} \mathrm{Zu}-$ dem wird eine Verstetigung und Weiterentwicklung der Forschungsförderung des ökologischen Landbaus als Maßnahme genannt, z.B. in Gestalt des „Bundesprogramm Ökologischer Landbau und andere Formen nachhaltiger Landwirtschaft" (BÖLN) ${ }^{37} .38$

\section{Naturschutzrelevante Maßnahmen des Sektors Landnutzung, Landnutzungsänderung und Forstwirtschaft (LULUCF)}

Neben der Landwirtschaft ist auch der LULUCF-Sektor aus Sicht von Naturschutz und Biodiversität bedeutsam. In diesem Sektor werden die THG-Emissionen zusammengefasst, die der Nutzung einer Fläche oder der Änderung der Flächennutzung zugerechnet werden. ${ }^{39} \mathrm{Da}-$ bei besteht die Besonderheit, dass durch Landnutzung nicht nur Emissionen reduziert werden können, sondern auch eine Einbindung von Kohlenstoff möglich ist, wie z. B. in Wäldern und Mooren (Senkenfunktion). Im Gegensatz zum Landwirtschaftssektor sind für den Bereich Landnutzung im KSG keine Jahresemissionsmengen festgelegt. Allerdings muss die Bilanz zwischen den THGEmissionen und dem Abbau von THG für die jeweiligen Landnutzungskategorien mindestens neutral sein (vgl. \$9 Abs. 1 S. 4 KSG). Im Jahr 2017 war der Sektor eine Senke (Einbindung von 15,1 Mio. t $\mathrm{CO}_{2}-\ddot{A} q$.), dazu trugen v. a. die Wälder bei (Speicherung von 57,6 Mio. $\mathrm{CO}_{2}-$ Äq.). ${ }^{40}$ Nach aktuellen Berechnungen des Thünen-Instituts sinkt die Kohlenstofffestlegung der Wälder bis zum Jahr 2020 allerdings voraussichtlich um ca. $80 \%$ auf 11,8 Mio. t $\mathrm{CO}_{2}$-Äq., was auf eine Verschiebung der Altersstruktur der Wälder und eine veränderte Holznutzung zurückgeführt wird. ${ }^{41}$ Das Ziel der Treibhausgasneutralität erfordert daher wirkungsvolle Maßnahmen. Neben der sinkenden Kohlenstofffestlegung in den Wäldern stellen dabei die Emissionen aus entwässerten Moorböden eine besondere Herausforderung dar. ${ }^{42} \mathrm{Zu}$ den aus Sicht von Naturschutz und Biodiversität relevanten Maßnahmenbereichen im Klimaschutzprogramm 2030 gehören der $\mathrm{Hu}-$ muserhalt bzw. Humusaufbau im Ackerland, der Erhalt von Dauergrünland, der Schutz von Moorböden sowie der Erhalt bzw. die nachhaltige Bewirtschaftung der Wälder. Als weiterer Maßnahmenbereich wird unter Verweis auf das 30 ha-Ziel der Bundesregierung ${ }^{43}$ die Eindämmung des Flächenverbrauchs für Siedlungs- und Verkehrszwecke genannt. ${ }^{44}$ Dieser Bereich ist allerdings nicht mit konkreten Maßnahmen untersetzt und wird daher im Folgenden nicht näher betrachtet.

\subsection{Humuserhalt und Humusaufbau im Ackerland}

Auf Grund der $\mathrm{CO}_{2}$-Speicherung im Humus kommt dem Humuserhalt und Humusaufbau im Ackerland klimaschützende Wirkung zu. Humus ist die Gesamtheit der abgestorbenen organischen Substanz im Boden. Es handelt sich hierbei um ein komplexes Gemisch von organischen Stoffen aus pflanzlicher, tierischer und mikrobieller Herkunft, das permanenten Ab-, Um- und Aufbauprozessen unterliegt. ${ }^{45} \mathrm{Maßnahmen}$ zum Humuserhalt und -aufbau haben zahlreiche positive Umweltwirkungen, insbesondere hinsichtlich des Schutzes der Biodiversität. ${ }^{46}$ So bietet der humose Oberboden Nahrungsquelle und zentralen Lebensraum für Bodenlebewesen. Eine ausreichende Humusversorgung ackerbaulich genutzter Böden sichert langfristig die Bodenfruchtbarkeit und beeinflusst wichtige ökologische Bodenfunktionen positiv. ${ }^{47}$ Das Klimaschutzprogramm sieht daher vor, das Kohlenstoffspeicherpotenzial der Böden verstärkt zu aktivieren. ${ }^{48}$ Entsprechende Maßnahmen sollen u.a. in der Ackerbaustrategie berücksichtigt werden, die derzeit erarbeitet wird. ${ }^{49}$ Im Einzelnen sind verschiedene Maßnahmen genannt, wie z.B. die Förderung von Fruchtfolgemaßnahmen für die Humusmehrung sowie eine humuserhaltende Bewirtschaftung in Betrieben, die bereits einen hohen Humusanteil auf ihren Flächen erreicht haben. Auch der Ausbau der Förderung von Gehölzstreifen, Feldgehölzen, Hecken, Knicks und Alleen sowie von Agroforstsystemen soll unterstützt werden. ${ }^{50}$ Als weitere Fördermaßnahmen werden die freiwillige Zertifizierung im Rahmen betrieblicher Audits von humusmehrenden Bewirtschaftungsmaßnahmen und die Entwicklung von Beratungswerkzeugen für die landwirtschaftliche Praxis zur schlaggenauen Bestimmung der Bodenkohlenstoffgehalte genannt. ${ }^{51}$ Über mögliche rechtliche Vorgaben im Rahmen der guten fachlichen Praxis soll nach Auswertung der zweiten Bodenzustandserhebung des Thünen-Instituts Mitte der 20er Jahre entschieden werden. ${ }^{52}$

31) Weingarten et al. (Fn. 6), S. 195

32) Klimaschutzprogramm 2030 (Fn. 5), S. 114 unter Verweis auf Weingarten et al. (Fn. 6), S. 195.

33) Klimaschutzprogramm 2030 (Fn. 5), S. 113.

34) Klimaschutzprogramm 2030 (Fn. 5), S. 113.

35) BMEL (Hrsg.), Zukunftsstrategie ökologischer Landbau. Impulse für mehr Nachhaltigkeit in Deutschland, 2. Aufl., Januar 2019.

36) Klimaschutzprogramm 2030 (Fn. 5), S. 113.

37) Näher hierzu BMEL, Bundesprogramm Ökologischer Landbau, https://www.bmel.de/DE/Landwirtschaft/Nachhaltige-Landnutzung/Oekolandbau/_Texte/BundesprogrammOekolandbau.html (Zugriff: 16.2.2020).

38) Klimaschutzprogramm 2030 (Fn. 5), S. 113.

39) Klimaschutzprogramm 2030 (Fn. 5), S. 124.

40) Klimaschutzprogramm 2030 (Fn. 5), S. 125.

41) Klimaschutzprogramm 2030 (Fn. 5), S. 126.

42) Klimaschutzprogramm 2030 (Fn. 5), S. 127.

43) Die Inanspruchnahme zusätzlicher Flächen für Siedlungs- und Verkehrszwecke soll bis zum Jahr 2030 auf unter 30 Hektar pro Tag begrenzt werden; Bundesregierung (Fn. 48), S. 159.

44) Klimaschutzprogramm 2030 (Fn. 5), S. 124.

45) Kolbe, Was ist Humus? https://www.bmel.de/DE/Landwirtschaft/Pflanzenbau/Boden/_Texte/Humus-Was-ist-das.html (Zugriff: 16.2.2020).

46) Klimaschutzprogramm 2030 (Fn. 5), S. 129f.

47) Bodennetzwerk, Warum ist Humus wichtig? https://www.bodenwelten.de/node/88 (Zugriff: 16.2.2020).

48) Klimaschutzprogramm 2030 (Fn. 5), S. 127.

49) Siehe hierzu das Diskussionspapier des BMEL (Hrsg.), „Ackerbaustrategie 2035. Perspektiven für einen produktiven und vielfältigen Pflanzenbau“, Dezember 2019.

50) Klimaschutzprogramm 2030 (Fn. 5), S. 128.

51) Klimaschutzprogramm 2030 (Fn. 5), S. 128.

52) Klimaschutzprogramm 2030 (Fn. 5), S. 127. 


\subsection{Erhalt von Dauergrünland}

Auch der Erhalt von Dauergrünland stellt eine Klimaschutzmaßnahme dar. ${ }^{33}$ Aufgrund der nicht stattfindenden Bodenbearbeitung und der hohen Kohlenstoffeinträge über Gräserwurzeln speichern Grünlandböden mehr Kohlenstoff als Ackerböden. ${ }^{54}$ Bei der Umwandlung von Dauergrünland in Ackerland wird organische Bodensubstanz mineralisiert und als $\mathrm{CO}_{2}$ in die Atmosphäre abgegeben. ${ }^{55} \mathrm{Zudem}$ wird Stickstoff freigesetzt. ${ }^{56}$ Die $\mathrm{CO}_{2}$-Freisetzung beim Umbruch von Dauergrünland verläuft sehr schnell und kann durch eine Neuschaffung von Grünland nur über sehr lange Zeiträume wieder aufgebaut werden. ${ }^{57}$ Deshalb sollte das existierende Dauergrünland möglichst flächenscharf erhalten werden. ${ }^{58} \mathrm{Da}$ Grünlandstandorte besonders artenreich sind, ${ }^{59}$ wirkt sich diese Maßnahme auch positiv auf den Natur- bzw. Biodiversitätsschutz aus. ${ }^{60}$ Insbesondere extensiv bewirtschaftetes Grünland ist ein wichtiger Standort für seltene artenreiche Pflanzengesellschaften, die an nährstoffarme Böden angepasst sind. ${ }^{61}$ Gleiches gilt für die an solche Standorte angepassten, zum Teil gefährdeten Tierarten. Darüber hinaus bietet Grünland zahlreiche Freizeit- und Erholungsmöglichkeiten und verfügt über einen hohen landschaftsästhetischen Wert. ${ }^{62}$

Im Klimaschutzprogramm ist eine Reihe von Maßnahmen zum Schutz von Dauergrünland genannt. ${ }^{63}$ So plant die Bundesregierung, ,die Regelungen zum Grünlanderhalt fortzuführen und eine Grünlandstrategie zur Sicherung und Stärkung einer dauerhaften Grünlandnutzung zu entwickeln". ${ }^{64}$ Insbesondere will das BMEL im Rahmen der Verhandlungen zur GAP nach 2020 dafür eintreten, „,dass auch künftig Regelungen für den Erhalt und Dauergrünland beibehalten werden“. ${ }^{65}$ Bisher erfolgt der Schutz von Dauergrünland im Rahmen der Greening-Anforderungen (1. Säule der GAP) gemäß Art. 43 ff. EU-Verordnung 1307/2013 ${ }^{66}$. Die darin geregelten Bewirtschaftungsanforderungen ${ }^{67}$ sind grundsätzlich für alle Empfänger der Basisprämie verbindlich. ${ }^{68} \mathrm{Nach}$ dem aktuellen Vorschlag der EU-Kommission zur GAP nach $2020^{69}$ ist der „Erhalt von Dauergrünland“ als guter landwirtschaftlicher und ökologischer Zustand (GLOZ) im Rahmen der einzelbetrieblichen Konditionalität geregelt. ${ }^{70} \mathrm{Er}$ entspricht grundsätzlich der bisherigen Greening-Anforderung. Allerdings ist noch mit Konkretisierungen durch delegierte Rechtsakte der EU-Kommission und/oder durch die Mitgliedstaaten in den nationalen Strategieplänen zu rechnen. ${ }^{71}$ In diesem Sinne werden im Klimaschutzprogramm im Hinblick auf die künftige nationale Umsetzung ${ }^{72}$,,verbindliche und möglich langfristig wirksame Regelungen zum Dauergrünlanderhalt" als GLÖZ-Standard angestrebt. ${ }^{73}$ Hierzu soll insbesondere die bisherige Greening-Komponente im Rahmen der künftigen Konditionalität ${ }^{74}$ und Ökoregelungen (,Eco-Schemes" ${ }^{75}$ der GAP nach 2020 weiterentwickelt werden. ${ }^{76}$ Genannt wird in diesem $\mathrm{Zu}-$ sammenhang z.B. eine geeignete Stichtagsregelung in der Vergangenheit. ${ }^{77}$ Bisher müssen die Mitgliedstaaten sicherstellen, dass der Anteil von Flächen mit Dauergrünland an der gesamten landwirtschaftlichen Fläche nicht um mehr als 5\% gegenüber dem Referenzjahr 2012 abnimmt (Art. 43 Abs. 2 EU-Verordnung 1307/2013). ${ }^{78}$ Der künftige GLÖZ-Standard soll im Rahmen der Nachfolgeregelung der AgarZahlVerpf $\mathrm{V}^{79}$ auf nationaler Ebene ,,ambitioniert und klimazielorientiert" umgesetzt werden. ${ }^{80}$ In diesem Zusammenhang wird u.a. ein Genehmigungsvorbehalt für Grünlandumwandlung und Grünlandumbruch, verbunden mit einer Pflicht zur Wiederanlage von Grünland genannt. ${ }^{81}$ Die Möglichkeit eines solchen Genehmigungsvorbehalts ist bisher in Art. 44 Abs. 1 EU-VO $639 / 2014^{82}$ vorgesehen und auf nationaler Ebene in $\$ 16$ Abs. 3 DirektZahlDurchfG ${ }^{83}$ geregelt. Auf kohlenstoffreichen Böden sollte laut Klimaschutzprogramm künftig ge- nerell keine Umwandlung erlaubt sein. ${ }^{84}$ Für die 2. Säule der GAP sieht das Klimaschutzprogramm eine Fortführung und Verbesserung einzelflächenbezogener und einzelbetrieblicher Fördermaßnahmen vor (z. B. im Hinblick auf eine geringere Stickstoff-Düngung und Bodenbearbeitung) ${ }^{85}$ Dies gilt auch für Agrarumweltmaßnahmen zur Förderung extensiver und an den Belangen des $\mathrm{Na}$ turschutzes orientierter Nutzungsformen sowie die Ausgleichszulage für benachteiligte Gebiete. Hierdurch soll die wirtschaftliche Attraktivität der Erhaltung des Dauergrünlands erhöht werden. ${ }^{86}$

53) Klimaschutzprogramm 2013 (Fn. 5), S. 130.

54) Weingarten et al. (Fn. 6), S. 155.

55) BfN, Grünland-Report, 2014, S. 6.

56) Weingarten et al. (Fn. 6), S. 155.

57) BfN (Fn. 55), S. 6; Weingarten et al. (Fn. 6), S. 155

58) Weingarten et al. (Fn. 6), S. 155.

59) BfN (Fn. 55), S. 5. Grünlandstandorte bieten Lebensraum für über die Hälfte aller in Deutschland vorkommenden Tier- und Pflanzenarten; UBA, Grünlandumbruch, https://www.umweltbundesamt.de/daten/land-forstwirtschaft/gruenlandumbruch\# gefahrdung-des-grunlands (Zugriff: 16.2.2020).

60) Vgl. Weingarten et al. (Fn. 6), S. 156; Möckel, NuR 2016, S. 741, 742 .

61) UBA (Fn. 59).

62) Möckel, (Fn. 60), 742.

63) S. hierzu Klimaschutzprogramm 2030 (Fn. 5), S. $130 \mathrm{ff}$.

64) Die Grünlandstrategie soll Angaben zur Bedeutung des Grünlands, Interessenkonflikten und Handlungsfelder zur Stärkung einer dauerhaften Grünlandnutzung benennen, Klimaschutzprogramm 2030 (Fn. 5), S. 131.

65) Klimaschutzprogramm 2030 (Fn. 5), S. 130.

66) AB1. EU, L 347, S. 608.

67) Neben dem Erhalt von Dauergrünland handelt es sich hierbei um die Anforderungen Anbaudiversifizierung und Mindestanteil an ökologischen Vorrangflächen.

68) Näher hierzu Möckel (Fn. 60), 744.

69) $\operatorname{COM}(2018) 392$ final.

70) Vgl. GLÖZ 1, Anhang III zum Strategieplan.

71) Grethe et al., Zur effektiven Gestaltung der Agrarumwelt- und Klimaschutzpolitik im Rahmen der Gemeinsamen Agrarpolitik der EU nach 2020, Stellungnahme, Mai 2019, S. 42.

72) Die konkrete Ausgestaltung der GLÖZ-Verpflichtungen soll stärker als bisher in der Verantwortlichkeit der Mitgliedstaaten liegen, Grethe et al. (Fn. 71), S. 42.

73) Klimaschutzprogramm 2030 (Fn. 5), S. 130.

74) Im Rahmen der Konditionalität müssen Empfänger von Direktzahlungen die „Grundanforderungen an die Betriebsführung“ (GAB) erfüllen und ihre Flächen in einem ,guten landwirtschaftlichen und ökologischen Zustand" (GLÖZ) halten (vgl. Art. 11 und 12 des Verordnungsentwurfs zur GAP nach 2020, $\operatorname{COM}(2018) 392$ final.

75) Bei den Ökoregelungen handelt es sich um ein neues Instrument der Agrarumwelt- und Klimapolitik (Grethe et al. (Fn. 71), S. 42). Hiermit sollen Betriebsinhaber unterstützt werden, die sich verpflichten, auf ihren förderfähigen Flächen „,dem Klimaund Umweltschutz förderliche Landbewirtschaftungsmethoden anzuwenden" (vgl. Art. 28 des Verordnungsentwurfs der EUKommission, COM(2018) 392 final).

76) Klimaschutzprogramm 2030 (Fn. 5), S. 130.

77) Klimaschutzprogramm 2030 (Fn. 5), S. 130.

78) Die Umsetzung in Deutschland erfolgt durch $₫ 16$ Abs. 1 und 2 DirektZahlVerpflG, näher hierzu Möckel (Fn. 60), $746 \mathrm{f}$.

79) Agrarzahlungen-Verpflichtungsverordnung v. 17.12.2014 (BAnz AT 23.12.2014 V1)

80) Klimaschutzprogramm 2030 (Fn. 5), S. 130.

81) Klimaschutzprogramm 2030 (Fn. 5), S. 130.

82) Delegierte Verordnung (EU) Nr. 639/2014 der Kommission vom 11. März 2014 zur Ergänzung der Verordnung (EU) Nr. 1307/2013, AB1. EU, L 181, S. 1.

83) Direktzahlungen-Durchführungsgesetz vom 9.7.2014 (BGB1. I S. 897).

84) Klimaschutzprogramm 2030 (Fn. 5), S. 132.

85) Klimaschutzprogramm 2030 (Fn. 5), S. 130.

86) Klimaschutzprogramm 2030 (Fn. 5), S. 131. 


\subsection{Schutz von Moorböden, Reduktion von Torfeinsatz in Kultursubstraten}

Ein weiterer Maßnahmenbereich des Klimaschutzes im LULUCF-Sektor betrifft den Schutz von Moorböden einschließlich der Reduzierung der Torfverwendung in Kultursubstraten. Moore entziehen der Atmosphäre $\mathrm{CO}_{2}$ und wirken damit als Kohlenstoffsenke. Das von den Pflanzen während ihres Wachstums aufgenommene $\mathrm{CO}_{2}$ wird nach ihrem Absterben im Torf festgelegt. Im Laufe der Zeit entwickeln sich Moore so zu einem bedeutenden Kohlenstoffspeicher. ${ }^{87}$ Auch aus Naturschutzsicht sind Moore sehr wertvoll, denn sie stellen einzigartige Zeugen der Landschaftsgeschichte dar und bieten Lebensraum für hoch spezialisierte Tiere und Pflanzen sowie Rast- und Brutstätte für viele Vögel. ${ }^{88}$ In der Vergangenheit wurden erhebliche Moorflächen entwässert, um diese landwirtschaftlich zu nutzen. Durch das Absenken der Wasserstände und die damit einhergehende Durchlüftung des Oberbodens wird die organische Substanz im Moorboden mineralisiert und in Form von $\mathrm{CO}_{2}$ an die Atmosphäre abgegeben. ${ }^{89}$ Entwässerte Moorböden sind daher eine bedeutende Quelle von THG-Emissionen. ${ }^{90}$ Eine Änderung der Bewirtschaftung (z.B. Umwandlung von Ackerland in Grünland oder eine Extensivierung der Grünlandnutzung), ggf. in Kombination mit einer saisonalen Anhebung des Wasserstandes, kann die Mineralisierung verlangsamen. Zur Beendigung des $\mathrm{CO}_{2}$-Ausstoßes ist eine vollständige Wiedervernässung der Flächen erforderlich. ${ }^{91}$ Durch den Abbau von Torf werden die Moore zerstört. ${ }^{92}$ Die Verwendung von Torf als Kultursubstrat verursacht THG-Emissionen infolge der Abtorfung, der Mineralisierung des Torfs und der nachfolgenden Nutzung der abgetorften Fläche. ${ }^{93}$ Die im Moor lebenden seltenen und vom Aussterben bedrohten Tier- und Pflanzenarten verlieren ihren Lebensraum.

Der Moorbodenschutz gilt daher sowohl als naturschutzals auch klimarelevante Maßnahme und soll laut Klimaschutzprogramm verstärkt gefördert werden. ${ }^{94}$ Hierzu strebt die Bundesregierung an, Anpassungen bestehender rechtlicher und förderrechtlicher Rahmenbedingungen mit dem Ziel vorzunehmen, ,einen möglichst effektiven Moorbodenschutz zu gewährleisten“. Insbesondere beabsichtigt die Bundesregierung, für den im aktuellen Vorschlag der EU-Kommission zur künftigen Gemeinsamen Agrarpolitik (GAP) vorgesehenen GLÖZ-Standard zu einem ,angemessenen Schutz von Feuchtgebieten und Torfflächen“95 sowie seiner ambitionierten Ausgestaltung einzutreten. ${ }^{96}$ Darüber hinaus sollen neue förderrechtliche Instrumente, einschließlich der dafür notwendigen Finanzierung für Programme zur dauerhaften Wiedervernässung von Moorböden, geschaffen werden. ${ }^{97}$ Auch eine Intensivierung von Forschungs- und Entwicklungsmaßnahmen ist geplant. Zur Reduktion des Torfeinsatzes in Kultursubstraten soll ein Bundesprogramm „Torfminderung“ aufgelegt werden, welches den Anbau und die Verwendung von Torfersatzstoffen durch finanzielle Förderung, Forschung, Beratung, Information und Schulung unterstützt. ${ }^{98}$ Diese Maßnahmen sollen im Freizeitgartenbau zu einem vollständigen Verzicht und im Erwerbsgartenbau zu einem weitgehenden Ersatz von Torf innerhalb der nächsten zehn Jahre führen. ${ }^{99}$

\subsection{Nachhaltige Bewirtschaftung der Wälder}

Ein wichtiger Maßnahmenbereich des Klimaschutzprogramms ist schließlich auch der „Erhalt und nachhaltige Bewirtschaftung der Wälder und Holzverwendung“.100 Wälder binden große $\mathrm{CO}_{2}$-Mengen. Dabei besteht die Besonderheit, dass Bäume langlebig sind und im Laufe ihres Wachstums der Atmosphäre fortwährend $\mathrm{CO}_{2}$ entziehen. ${ }^{101}$ Auch die Waldböden können über lange Zeiträume Kohlenstoffvorräte aufbauen bzw. konservieren. ${ }^{102}$ Halten sich Holzzuwachs und Holzeinschlag die Waage, bleibt der
Kohlenstoffspeicher in der Biomasse konstant (vorausgesetzt, der Wald ist hinsichtlich seiner Alters- bzw. Durchmesserstruktur und Baumartenzusammensetzung nachhaltig aufgebaut). Auch die stoffliche Nutzung des geernteten Holzes leistet einen Beitrag zur Reduzierung der $\mathrm{CO}_{2}$ Konzentration in der Atmosphäre, da der gebundene Kohlenstoff in den Holzprodukten über eine gewisse Zeit erhalten bleibt. Hinzu kommen stoffliche und energetische Substitutionseffekte. ${ }^{103}$ Besonders günstig wirkt die Kaskadennutzung, d.h. die aufeinander folgende, mehrfache Verwertung desselben Holzes zur Herstellung von Materialien, gefolgt von einer abschließenden energetischen Nutzung. ${ }^{104}$ Die aus Klimaschutzgründen erforderliche nachhaltige Bewirtschaftung der Wälder ist auch vorteilhaft für den Naturschutz, denn der Wald ist Lebensraum für spezifische Waldbiotope und Arten, die von einer nachhaltigen Bewirtschaftung profitieren. ${ }^{105}$

Die im Klimaschutzprogramm genannten Maßnahmen zur nachhaltigen Bewirtschaftung der Wälder und zur Holznutzung sind vielfältig und tragen auch den aktuellen extremwetterbedingten Waldschäden Rechnung. Vorgesehen ist z.B. eine verstärkte Förderung von Maßnahmen zur Entwicklung von klimatoleranten Mischwäldern über die Gemeinschaftsaufgabe GAK. ${ }^{106}$ Ziel ist eine Wiederbewaldung der Schadflächen und verstärkte Anpassung der Wälder an den Klimawandel unter Beachtung ökologischer Belange. Hinzu kommen flankierende Maßnahmen wie z. B. ein optimiertes Monitoring und Wildtiermanagement sowie eine verstärkte internationale Zusammenarbeit zur Sicherstellung einer nachhaltigen Waldbewirtschaftung weltweit. Weiterhin genannt sind eine verstärkte Förderung von F\&E-Vorhaben, Fach- und Verbraucherinformationen, Ideenwettbewerben und Modellvorhaben mit dem Ziel einer klimafreundlichen Holzverwendung und der Förderung von klimabewusstem Verbraucherverhalten. ${ }^{107}$

87) BfN, Ökosystemleistungen der Moore, https://www.bfn.de/ themen/biotop-und-landschaftsschutz/moorschutz/oekosystemleistungen.html (Zugriff: 16.2.2020).

88) BUND Naturschutz, Lebensraum Moor, https://www.bundnaturschutz.de/natur-und-landschaft/moore-in-bayern/lebensraum-moor.html (Zugriff: 16.2.2020).

89) Weingarten et al. (Fn. 6), S. 144

90) Klimaschutzprogramm 2030 (Fn. 5), S. 133

91) Aufgrund der daraufhin einsetzenden Methanfreisetzungen dauert es einige Zeit, bis die Klimabilanz wieder ausgeglichen ist; Weingarten et al. (Fn. 6), S. 145.

92) Greifswald Moor Centrum, Moorwissen; https://www.moorwissen.de/de/moore/moornutzung/nutzungsarten.php (Zugriff: 16.2 .2020$)$.

93) Weingarten et al. (Fn. 6), S. 152

94) Klimaschutzprogramm 2030 (Fn. 5), S. 133

95) S. hierzu COM/2018/392 final, Anhang III, GLÖZ 2.

96) Die EU-Kommission definiert im Anhang III zum Verordnungsentwurf für den Strategieplan (COM(2018) 392 final) 16 Grundanforderungen an die Betriebsführung (GAB) und 10 Standards für den guten landwirtschaftlichen und ökologischen Zustand von Flächen (GLÖZ). Landwirte, die Direktzahlungen erhalten, müssen demnach im Vergleich zu den derzeit geltenden Standards (Cross Compliance und Greening) künftig weitere Voraussetzungen erfüllen (Grethe et al. (Fn. 71), S. 42).

97) Klimaschutzprogramm 2030 (Fn. 5), S. 133

98) Siehe hierzu im Einzelnen Klimaschutzprogramm 2030 (Fn. 5), S. 133.

99) Klimaschutzprogramm 2030 (Fn. 5), S. 134.

100) Klimaschutzprogramm 2030 (Fn. 5), S. $135 \mathrm{ff}$

101) Weingarten et al. (Fn. 6), S. 24.

102) Weingarten et al. (Fn. 6), S. 24.

103) Näher hierzu Weingarten et al. (Fn. 6), S. 24.

104) Weingarten et al. (Fn. 6), S. 24

105) Klimaschutzprogramm 2030 (Fn. 5), S. 136.

106) Z.B. Vertragsnaturschutz zur Verbesserung des $\mathrm{CO}_{2}$-Speicherpotenzials oder des Strukturreichtums des Waldes.

107) Klimaschutzprogramm 2030 (Fn. 5), S. 136 


\section{Zusammenfassende Bewertung}

Eine nachhaltige Landnutzung bzw. Bewirtschaftung landund forstwirtschaftlicher Flächen stellt eine entscheidende Brücke zwischen Klimaschutz und Erhalt der biologischen Vielfalt dar. Diesbezüglich ergeben sich aus dem neuen Klimaschutzrecht vielversprechende Ansätze aus Sicht des Naturschutzes. Die Realisierung entsprechender Potenziale hängt allerdings entscheidend von den getroffenen Klimaschutzmaßnahmen ab. Das Klimaschutzprogramm listet in den Handlungsfeldern eine breite Palette von Maßnahmen auf. Genannt werden v. a. konzeptionelle und weiche Instrumente wie die Erarbeitung von Strategien, finanzielle Anreize, Forschung, Ideenwettbewerbe, Modellprojekte, Beratung und Information. Im Mittelpunkt steht die erhoffte Steuerungswirkung durch Fördermittel; erste Finanzierungsmaßnahmen wurden bereits im Rahmen des Klimapakets verabschiedet (z. B. auch im Bereich der Landwirtschaft). Ordnungsrechtliche Instrumente (Verbote, Beschränkungen) sind im Klimaschutzprogramm hingegen kaum zu finden. Eine Konkretisierung bzw. nähere Ausführung der Maßnahmen gegenüber den Angaben des Klimaschutzplans ist nur an wenigen Stellen zu erkennen. Häufig wird auf bereits vorliegende Strategien und Programme verwiesen; neue Instrumente werden kaum in Aussicht gestellt. Eine Ausnahme bildet die angekündigte Verschärfung des Düngerechts, diese erfolgt allerdings nicht primär aus Gründen des Klimaschutzrechts, sondern im Hinblick auf die Verurteilung Deutschlands wegen mangelnder Umsetzung der Nitrat-RL sowie dem diesbezüglich laufenden Vertragsverletzungsverfahren. Im Bereich der GAP sind keine wesentlichen Verbesserungen zu erwarten, hier geht es vor allem darum, das derzeitige Schutzniveau $\mathrm{zu}$ halten (vgl. Grünlanderhalt). Gerade in diesem Bereich bestünde allerdings erhebliches Potenzial, durch angepasste Bewirtschaftungsformen sowohl dem Klimaschutz als auch der Biodiversität zur Durchsetzung zu verhelfen. Weitere Möglichkeiten zur Reduktion von Treibhausgasen könnte der Bereich des Flächensparens bieten, allerdings werden insofern keine konkreten Maßnahmen ausgeführt. Unklar ist auch, wie die verstärkte internationale Zusammenarbeit zur Sicherstellung einer nachhaltigen Waldbewirtschaftung erreicht werden soll. Nicht zuletzt im wichtigen Handlungsfeld des Moorschutzes bleibt offen, wie ein ambitioniertes Schutzniveau ausgestaltet sein könnte und wie z.B. die notwendigen Veränderungen im Bereich der Torfverwendung ohne konkrete rechtliche Vorgaben tatsächlich erreicht werden können. Es bleibt zu hoffen, dass im Rahmen der erforderlichen fachgesetzlichen Konkretisierung für diese Fragen wirkungsvolle Lösungen gefunden werden. Ohne instrumentelle Nachjustierungen werden weder die Klimaschutzziele noch die Synergieeffekte für den $\mathrm{Na}-$ turschutz im angestrebten Umfang zu erreichen sein.

Open Access. Dieser Artikel wird unter der Creative Commons Namensnennung 4.0 International Lizenz veröffentlicht, welche die Nutzung, Vervielfältigung, Bearbeitung, Verbreitung und Wiedergabe in jeglichem Medium und Format erlaubt, sofern Sie den/die ursprünglichen Autor(en) und die Quelle ordnungsgemäß nennen, einen Link zur Creative Commons Lizenz beifügen und angeben, ob Änderungen vorgenommen wurden.

Die in diesem Artikel enthaltenen Bilder und sonstiges Drittmaterial unterliegen ebenfalls der genannten Creative Commons Lizenz, sofern sich aus der Abbildungslegende nichts anderes ergibt. Sofern das betreffende Material nicht unter der genannten Creative Commons Lizenz steht und die betreffende Handlung nicht nach gesetzlichen Vorschriften erlaubt ist, ist für die oben aufgeführten Weiterverwendungen des Materials die Einwilligung des jeweiligen Rechteinhabers einzuholen. Weitere Details zur Lizenz entnehmen Sie bitte der Lizenzinformation auf http://creativecommons.org/licenses/by/4.0/deed.de.

Open access funding provided by Projekt DEAL.

\section{Das Gutachten des Amtsveterinärs im Tierschutzrecht ${ }^{\star}$}

\section{Matthias Hettich}

๑) Springer-Verlag GmbH Deutschland, ein Teil von Springer Nature 2020

Dem Gutachten des beamteten Tierarztes kommt in der tierschutzrechtlichen Praxis eine zentrale Stellung zu. In der Regel ist es die gesetzliche Voraussetzung dafür, dass dem Tierhalter ein Tier wegen Verletzung der Anforderungen an Ernährung, Pflege oder Unterbringung fortgenommen werden kann. Diese große Bedeutung des Gutachtens des Amtsveterinärs steht im Missverhältnis zu der geringen rechtlichen Durchdringung der damit verbundenen Fragen. Welche formalen und inhaltichen Anforderungen an das Gutachten des beamteten Tierarztes zu stellen sind, ist bisher nur in Ansätzen geklärt.

\section{Einleitung: Gesetzliche Ausgangslage}

Eine Fortnahmeverfügung wegen tierschutzwidriger $\mathrm{Zu}-$ stände nach $\$ 16$ a Abs. 1 S. 2 Nr. 2 HS 1 Tierschutzgesetz

Matthias Hettich,

Richter am VGH Baden-Württemberg,

Lehrbeauftragter an der Universität Mannheim,

Mannheim, Deutschland
(TierSchG) ist eine einschneidende Maßnahme. Sie enthält die Anordnung an den Halter, das Tier herauszugeben, und führt für diesen - bei der nach Landesrecht vorzunehmenden Vollstreckung - zum Verlust des Besitzes am Tier. ${ }^{1}$ Ihr kann zudem die Veräußerung des fortgenommenen Tieres durch die Tierschutzbehörde nach $\$ 16$ a Abs. 1 S. 2 Nr. 2 HS 2 TierSchG und damit der endgültige Eigentumsverlust für den Halter und Eigentümer nachfolgen. ${ }^{2}$ Die Fortnahme setzt schwerwiegende tierschutzwidrige Zustände voraus. Sie kann nur ausgesprochen werden, wenn ein Tier mangels Erfüllung der Anforderungen des $₫ 2$ TierSchG erheblich vernachlässigt ist oder schwerwiegende Verhaltensstörungen aufweist. Zudem verlangt $\$ 16$ a Abs. 1 S. 2 Nr. 2

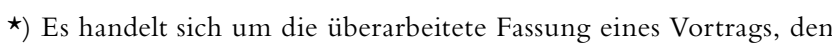
der Verfasser beim 2. Arbeitsgespräch für Amtsveterinäre und Juristen der Landesbeauftragten für Tierschutz am 26.9.2019 in Stuttgart gehalten hat.

1) Grundlegend: BVerwG, Urt. v. 12.1.2012 - 7 C 5.11, BVerwGE $141,311,317 \mathrm{ff}$.

2) Dazu: Ludwig, NuR 2014, 821. 